This is an electronic reprint of the original article. This reprint may differ from the original in pagination and typographic detail.

Author(s): Kähärä, Topi; Tallinen, Tuomas; Timonen, Jussi

Title: $\quad$ Numerical model for the shear rheology of two-dimensional wet foams with deformable bubbles

Year: $\quad 2014$

Version:

Please cite the original version:

Kähärä, T., Tallinen, T., \& Timonen, J. (2014). Numerical model for the shear rheology of two-dimensional wet foams with deformable bubbles. Physical Review E, 90(3), Article 032307. https://doi.org/10.1103/PhysRevE.90.032307

All material supplied via JYX is protected by copyright and other intellectual property rights, and duplication or sale of all or part of any of the repository collections is not permitted, except that material may be duplicated by you for your research use or educational purposes in electronic or print form. You must obtain permission for any other use. Electronic or print copies may not be offered, whether for sale or otherwise to anyone who is not an authorised user. 


\title{
Numerical model for the shear rheology of two-dimensional wet foams with deformable bubbles
}

\author{
T. Kähärä* and T. Tallinen \\ Department of Physics, University of Jyväskylä, Finland \\ J. Timonen \\ Department of Physics and Nanoscience Center, University of Jyväskylä, Finland \\ and ITMO University, Kronverkskii ave. 49, 197101, Saint Petersburg, Russia
}

(Received 16 May 2014; published 23 September 2014)

\begin{abstract}
Shearing of two-dimensional wet foam is simulated using an introduced numerical model, and results are compared to those of experiments. This model features realistically deformable bubbles, which distinguishes it from previously used models for wet foam. The internal bubble dynamics and their contact interactions are also separated in the model, making it possible to investigate the effects of the related microscale properties of the model on the macroscale phenomena. Validity of model assumptions was proved here by agreement between the simulated and measured Herschel-Bulkley rheology, and shear-induced relaxation times. This model also suggests a relationship between the shear stress and normal stress as well as between the average degree of bubble deformation and applied shear stress. It can also be used to analyze suspensions of bubbles and solid particles, an extension not considered in this work.
\end{abstract}

DOI: 10.1103/PhysRevE.90.032307

PACS number(s): 83.80.Iz, 47.57.Bc, 83.60.Fg

\section{INTRODUCTION}

Understanding the rheological properties of flowing liquid foams, and those of soft matter in more general, is relevant in many technological applications that range from microscale flows (of, e.g., biological and medical applications) to industrial-scale processes such as manufacturing of fiber-based products. Many properties of such systems can be determined experimentally, but we would also like to understand theoretically the associated phenomena. Because of the complicated nature of viscoelastic flows, there does not exist a general mathematical framework for a comprehensive description of such flows. It is thus natural to resort to numerical simulations for that purpose.

Several models for foam-like materials already exist, and a wide range of phenomena have been studied both experimentally and by simulations, see for example [1-74]. Most of the previous foam models have, however, assumed a dry foam, i.e., foam with a low-liquid fraction, and by now the physics of dry foams is quite well understood. In spite of their technological importance, mainly because they are more difficult to model, much less is known about wet foams. Rheology of dry foams is mostly determined by (quasistatic) minimization of surface energy, though viscous effects cannot be completely excluded $[5,15]$. In contrast with this, rheology of wet foams arises predominantly from an interplay of surface tension and viscous drag forces. Wet foams typically have a liquid fraction, $\phi$, of a few percent so that in an unperturbed state their bubbles are still closely packed and jammed.

We introduce here a numerical model for quasi-twodimensional wet foam, which we will call in the following the Dynamic Soft Matter and Liquid (DySMaL) model. The bubbles of this model are deformable, which distinguishes it from the previous disk-foam models [1], and allows us to analyze bubble deformations that are prominent at high-shear

*Corresponding author: topi.kahara@jyu.fi rates. This deformability also allows the possibility of modeling, e.g., narrow channels where bubbles are significantly elongated. In addition, the model clearly separates the internal dynamics of bubbles from their contact dynamics. For example in the disk model these are not really separated but rather described by simplified forces between bubble centers of mass. In the DySMaL model the emphasis is on describing the bubble interfaces in greater detail and contacts happen between these interfaces and not between bubble centers of mass. This separation of forces also makes it possible to modify either the internal dynamics of the suspended object or the contact model independently and thus gain insight on how these microscopic changes affect the rheological properties of a macroscopic system. An example of this would be to replace the (deformable) bubbles with (non-deformable) solid spheres or other shapes, while keeping the contact model unchanged. This also opens up the possibility of modeling mixtures of solids and bubbles, as required by certain technical applications, which is one of the key motivations for developing the model. Contacts between bubbles are modeled essentially as simple viscous friction, an idea somewhat analogous with the viscous froth model [15], see Sec. II for details. Still, the viscous froth model is essentially a model for dry foam and as such not really comparable, although it might be possible to push the DySMaL model into the sub-percent liquid fraction regime and model a dryish foam. In addition, the contact dynamics of the DySMaL model could also be modified to account for a completely dry friction or a more detailed model of the fluid which mediates the viscous forces between suspended objects. However, these are avenues to be explored in the future and not directly relevant to this work.

In this paper we compare the DySMaL model to experimental results and our main objective is to test the validity of the model in a specific setup. We find it essential that the model is able to reproduce relatively simple experimental results with reasonable accuracy before making any inferences on how microscopic changes affect the macroscale behavior 
of the system or using the model to simulate even more complex systems. As a model system, we study a sheared monolayer of foam, for which recent experimental data are readily available $[10,14,17,27,28,44,49,52-54]$, and which already allows the assessment of several important features of two-dimensional foam rheology.

The outline of the paper is as follows. In Sec. II we introduce the DySMaL model. Simulations of a sheared monolayer of foam are presented in Sec. III together with comparison with experimental results. Finally, a summary of the results obtained is given and future developments of the model are discussed in Sec. IV. In addition, there are two appendices in which results of the model are compared against known analytical results.

\section{THE MODEL}

\section{A. Overview}

The (DySMaL) model describes objects that are aggregates of linked particles (see Fig. 1 for an illustration) and are suspended in a carrier fluid. It is thus a particle-based (Lagrangian) model in which particles propagate in time based on Newtonian dynamics. The carrier fluid, described by its density, viscosity, and pressure, is not simulated explicitly, but is accounted for in an implicit fashion through viscous interactions of the suspended objects. This description of the carrier fluid is reasonable for wet foams in which fluid conforms to the motion of the bubbles. A similar approach has also been adopted in other models described, e.g., in [1,15,16,41,47].

We consider here a two-dimensional (2D) foam constrained between walls. This foam is composed of individual deformable bubbles whose surfaces are discretized into $N$ segments. Although the dynamics of the system is constrained into $2 \mathrm{D}$, there is a fixed depth in the system also in the third dimension. Since a closed loop of surface segments forms a

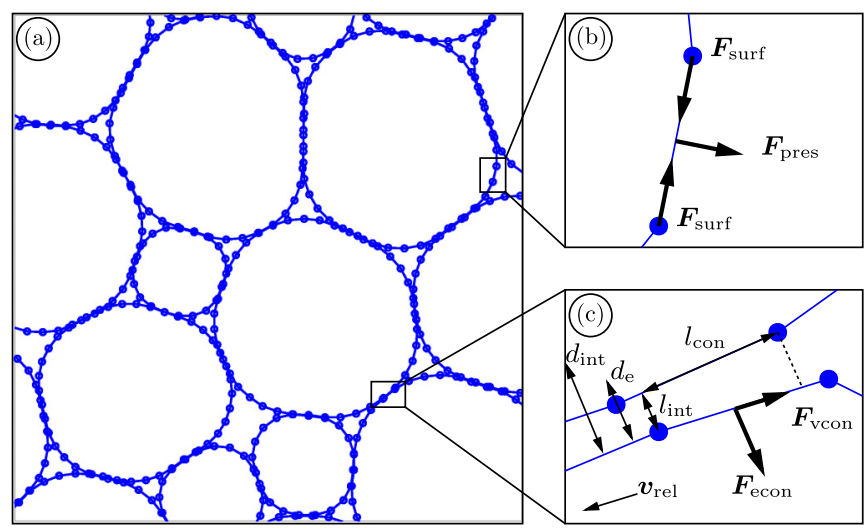

FIG. 1. (Color online) A deformable bubble model for wet foams. (a) Bubbles are modeled as segments and nodes with dynamics resulting from pressure differences, surface tension, and interactions with other bubbles. The pressure and surface tension forces are applied for each segment as illustrated in (b). Viscous $\left(\boldsymbol{F}_{\mathrm{vcon}}\right)$ and elastic $\left(\boldsymbol{F}_{\text {econ }}\right)$ contact forces between two bubbles surfaces moving past each other with relative velocity $\boldsymbol{v}_{\text {rel }}$ are illustrated for one segment in (c). The viscous and elastic contact ranges, $d_{i n t}$ and $d_{e}$, respectively, and the shortest distance $l_{\text {int }}$ between the segments and the segment overlap $l_{\text {con }}$ are marked. bubble, it is actually modeled as a cylinder of fixed depth. It has a volume and surface area calculable from the positions and lengths of the surface segments, and an internal pressure, $\left(p_{\text {in }}\right)$. We assume that bubbles are at constant temperature and that matter is not exchanged between the insides of bubbles and the carrier fluid. The internal pressure of bubbles thus obeys the ideal gas law,

$$
p_{\text {in }} V=w,
$$

where $w$ is a bubble-specific constant.

Naturally bubble surfaces also move and deform under surface tension and viscous drag forces. In the model, these forces are determined segment-wise, and then distributed to the ends of the segments, which are treated as point particles by the time integrator.

\section{B. Forces and dynamics}

For a single bubble there are two forces, surface tension and pressure, which are mainly responsible for its dynamics. The surface-tension force acting on an endpoint of a segment is given by

$$
\boldsymbol{F}_{\text {surf }}=\sigma \frac{l_{\text {seg }}}{l_{\text {ave }}} d_{\mathrm{z}} \hat{l}_{\text {seg }}
$$

where $\sigma$ is the surface tension, $l_{\text {seg }}$ is the length of the segment, $l_{\text {ave }}$ is the average length of segments at the same surface, $d_{\mathrm{z}}$ is the depth of the third dimension, and $\hat{l}_{\text {seg }}$ is a unit vector pointing towards the other end of the segment. We note that the factor $l_{\text {seg }} / l_{\text {ave }}$ ensures approximately even segment lengths, and is included solely so as to have a numerically stable surface discretization.

The pressure force on a segment of a bubble surface is determined from the expression

$$
\boldsymbol{F}_{\text {pres }}=\left(p_{\text {in }}-p_{\text {out }}\right) l_{\text {seg }} d_{\mathrm{z}} \hat{\boldsymbol{n}}_{\text {seg }},
$$

in which $p_{\text {in }}$ is the internal pressure of the bubble, $p_{\text {out }}$ is the pressure of the carrier fluid at the center of a segment, $l_{\text {seg }}$ is its length, and $\hat{\boldsymbol{n}}_{\text {seg }}$ is its outward unit normal vector. Value for the external pressure, $p_{\text {out }}$, is obtained from a predefined pressure field, while the internal pressure $p_{\text {in }}$ is obtained using Eq. (1).

Balance between the surface tension and pressure should lead to Laplace's law, and the net pressure on a bubble must produce the correct buoyancy equation. These requirements are shown to be fulfilled by the model in Appendices A and B.

On the foam scale also interactions between bubbles have to be modeled. To this end, we employ viscous and elastic contact forces. The viscous contact force is determined by the expression

$$
\boldsymbol{F}_{\mathrm{vcon}}=-\mu \frac{\boldsymbol{v}_{\mathrm{rel}}}{l_{\mathrm{int}}} l_{\mathrm{con}} d_{\mathrm{z}},
$$

where $\mu$ is the viscosity of the carrier fluid, $l_{\text {con }}$ is the length of the segment overlap, and $l_{\text {int }}$ is the shortest distance between the segments, see Fig. 1 for illustration. The basic idea is that the two interacting segments are treated as plates with an overlapping area of $l_{\text {con }} d_{\mathrm{z}}$ separated by a layer of carrier fluid which mediates a viscous force that is directly proportional to the shear rate $v_{\text {rel }} / l_{\text {int }}$. Computation of the viscous force is 
cut off at a distance $\left(d_{\text {int }}\right)$ larger than the typical liquid-film thickness between the bubbles.

In order to ensure structural integrity and prevent penetration of the bubbles, we apply a repulsive elastic contact force for segments,

$$
\boldsymbol{F}_{\text {econ }}=-k_{\mathrm{e}} l_{\text {con }} d_{\mathrm{z}}\left(1-l_{\mathrm{int}} / d_{\mathrm{e}}\right) \hat{l}_{\mathrm{int}},
$$

when $l_{\text {int }}$ is less than the contact range $d_{e}\left(d_{e} \ll d\right.$ and $\left.d_{e}<d_{\text {int }}\right)$. Here $k_{\mathrm{e}}$ is the contact modulus and $\hat{l}_{\text {int }}$ is a unit vector towards the closest point in the opposing segment. To prevent any surface-tension and pressure-related forces causing penetration, we require that $k_{e} \gg \sigma / d$ and $k_{e} \gtrsim p_{\text {out }}$. Like all forces in the model, $\boldsymbol{F}_{\text {econ }}$ is first determined for segments and then distributed to the endpoints of the segments.

We use explicit time integration to solve Newton's equations of motion of the points that connect the segments. Each point has a mass, $m=\rho d_{e} l_{\text {seg }} d_{\mathrm{z}}$, where $\rho$ is the density of the carrier liquid. To determine a timestep, $\Delta t$, it is useful to consider the elastic oscillation time, $t_{e} \approx \pi \sqrt{\frac{\rho d_{e}^{2}}{k_{e}}}$, and the inverse friction time, $t_{v} \approx \frac{\rho d_{e}^{2}}{\mu}$, of the bubble-bubble interactions. Numerical stability requires $\Delta t<\min \left(t_{e}, t_{v}\right)$.

\section{Parameter setup}

Here we use the model to study a similar foam as studied in the experiments reported in [44,49,52-54], hence the properties of the carrier fluid were taken directly from them, and are shown in Table I. We consider a bidisperse foam with two bubble diameters, $\left\langle d_{1}\right\rangle=2 \mathrm{~mm}$ and $\left\langle d_{2}\right\rangle=4 \mathrm{~mm}$, and a monodisperse foam with an average bubble diameter of $\langle d\rangle=3$ $\mathrm{mm}$. Thus, both foams have the same average bubble diameter, all bubbles included. Two randomly generated samples of both foams were used, and a $10 \%$ uniform variation in the bubble diameter was included. The total number of bubbles in the systems considered was between 1520 and 1700, and the liquid fraction was $\approx 0.07$.

Foam was constrained between two walls $8 \mathrm{~cm}$ apart, and in the direction parallel to the walls the system was $16 \mathrm{~cm}$ long with periodic boundary conditions, see Fig. 2 for snapshots of foam configurations. The depth of the third dimension was set to $d_{\mathrm{z}}=2 \mathrm{~mm}$. In the experiments [44,49], a monolayer foam had been confined between the bulk liquid and a top plate that exerted additional drag to the bubbles. It would be straightforward to include such a drag in the model, but we ignore it here since our focus is in the dynamics that arises from bubble-bubble interactions. In this respect, simulated system corresponds to a shallow cross section of a deep layer of foam, whose velocity varies only in the two planar dimensions. All forces in the model are directly proportional to $d_{z}$, i.e., the

TABLE I. Fluid properties.

\begin{tabular}{lccc}
\hline \hline Parameter & Symbol & Value & Unit \\
\hline Density & $\rho$ & 1000 & $\mathrm{~kg} / \mathrm{m}^{3}$ \\
Dynamic viscosity & $\mu$ & 1.8 & $\mathrm{mPa} \mathrm{s}$ \\
Surface tension & $\sigma$ & 28 & $\mathrm{mN} / \mathrm{m}$ \\
Pressure & $p$ & 103 & $\mathrm{kPa}$ \\
\hline \hline
\end{tabular}

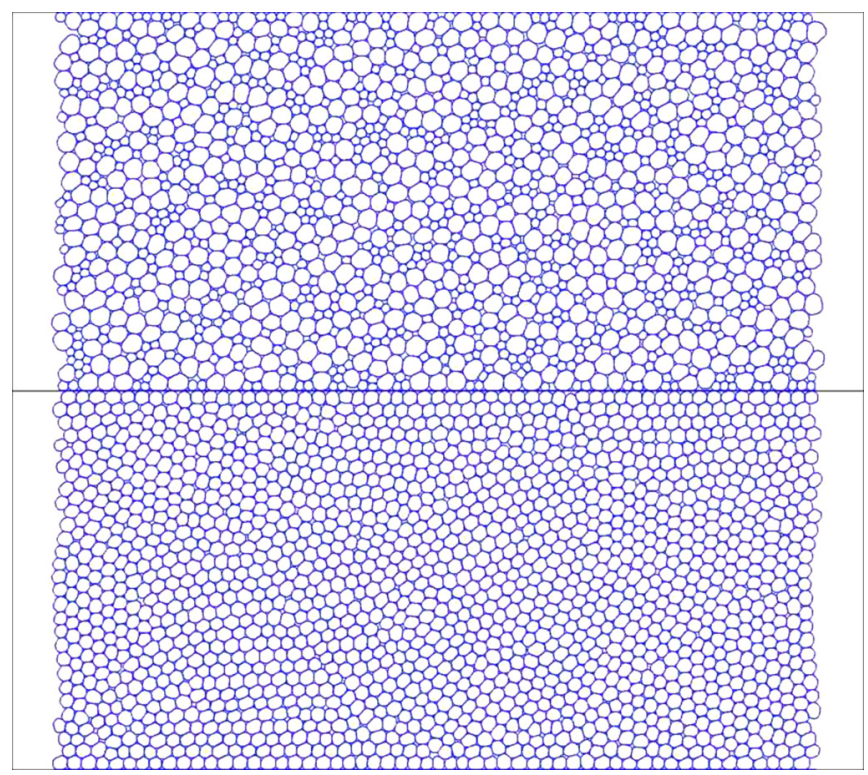

FIG. 2. (Color online) A snapshot of the simulated foam geometry. Top panel: bidisperse foam and bottom panel: monodisperse foam. For an animated version, see Supplemental Material [75].

results do not depend on it, but we include it for dimensional consistency.

Over the range of simulated shear rates (up to $\dot{\gamma} \approx 1.7 \frac{1}{\mathrm{~s}}$ ), flow can be considered non-inertial, and the relevant dimensionless parameter that varies with the shear rate is the capillary number $C a=\frac{\mu\langle d\rangle \dot{\gamma}}{\sigma}$ which is $\approx 4 \times 10^{-4}$ for the highest shear rates. Another dimensionless quantity to point out is the hydrostatic pressure over surface tension, $\frac{p_{\text {out }}\langle d\rangle}{\sigma} \approx 10^{4}$. Such a high value implies that bubbles are essentially incompressible under the surface tension.

For numerical implementation the bubbles are discretized to 20 segments (small bubbles) or 40 segments (large bubbles). The parameters $d_{\text {int }}, d_{e}$, and $k_{e}$ in the computation of force and the simulation timestep $\Delta t$ are closely related to the numerical implementation and as such have limited physical relevance, however there are a few physical considerations in addition to the numerical requirements mentioned above.

Since in this particular case the assumption is that the film of carrier fluid between the bubble surfaces is (virtually) incompressible, the contact modulus $k_{e}$ needs to be large enough to effectively ensure this. Ideally one would set $k_{e}$ to infinity but numerically this is unfeasible because of the numerical stability requirements. Since the bubbles are already nearly incompressible, it is sufficient that the compressibility of the film is of the same magnitude. The value $k_{e}=10^{5} \mathrm{~Pa}$ meets these requirements.

The film thickness $d_{e}$ can be estimated theoretically as a function of local shear rate [19]. Since we use a single value everywhere in the system we have used the average global shear rate as a guide and found that $d_{e}=10 \mu \mathrm{m}$ is of the correct order of magnitude. In addition, we have been made aware that recent optical coherence tomography measurements for foam flows exhibit slip layers thicknesses of similar magnitude [76].

The interaction distance $d_{\text {int }}$ is basically a cutoff beyond which interactions are not modeled and is included to reduce 
computational load. In this work the modeled system is quite dense and the average contact distances will be very close to the film thickness $d_{e}$ thus a value $d_{\text {int }}=20 \mu \mathrm{m}$, double the film thickness, is a reasonable choice. This value should be increased significantly only for considerably sparser systems with large bubble separations compared to the film thickness. However, in such a case the current contact model would probably be an inadequate approximation anyway.

Finally, based on the numerical stability considerations, we set the timestep $\Delta t=1 \mu$ s that is over two orders of magnitude smaller than the bubble relaxation time $\mu\langle d\rangle / \sigma \approx 200 \mu \mathrm{s}$ [1]. Simulations in this work represent up to $50 \mathrm{~s}$ of real time, corresponding to 50 million time steps.

\section{SIMULATION RESULTS}

\section{A. Herschel-Bulkley constitutive equation}

We start by comparing results of our shear simulations with the Herschel-Bulkley constitutive equation

$$
\tau=\tau_{Y}+k \dot{\gamma}^{\beta}
$$

where $\tau$ is the shear stress, $\tau_{Y}$ is the yield stress, $k$ is the viscous component of stress, $\dot{\gamma}$ is the shear rate, and $\beta$ is the power-law exponent. In order to determine all these coefficients we ran several simulations with the walls of the system moving in opposite directions with equal speeds, which in turn created a velocity gradient in the foam. After the system had reached a steady state, we extracted the velocity gradient from the foam and the forces acting on the walls as time averages. Without a wall drag the velocity gradient was linear, and we could do a direct fit of the simulated $\tau(\dot{\gamma})$ behavior by the Herschel-Bulkley equation, or rather its inverse,

$$
\dot{\gamma}=\max \left(0, \frac{\tau-\tau_{Y}}{k}\right)^{1 / \beta} .
$$

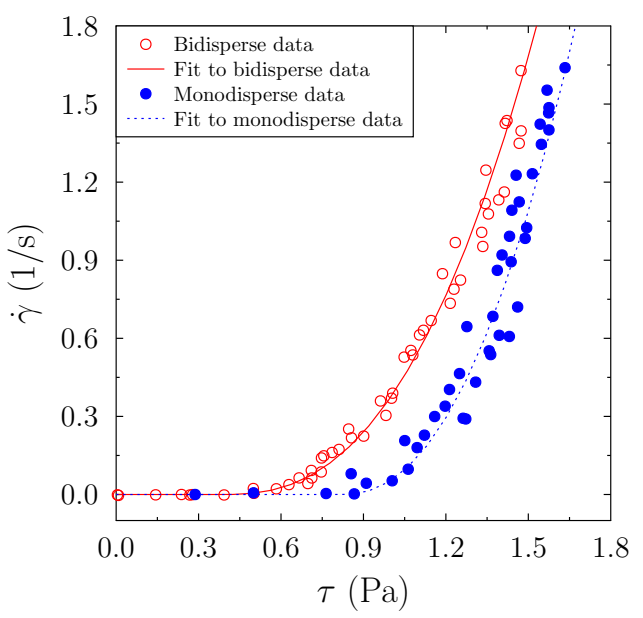

FIG. 3. (Color online) Shear rate as a function of shear stress for a bidisperse and monodisperse foam. The full and dotted lines fitted to the simulation results (open and filled spheres, respectively) correspond to inverses of the Herschel-Bulkley equation $\dot{\gamma}=\max \left[0,\left(\tau-\tau_{Y}\right) / k\right]^{1 / \beta}$ with coefficients given in Table II.
TABLE II. Best least-squares-fit parameters for the HerschelBulkley constitutive equation, $\tau=\tau_{Y}+k \dot{\gamma}^{\beta}$, for a bidisperse and monodisperse foam. The errors given correspond to the $1 \sigma$ confidence level.

\begin{tabular}{lccc}
\hline \hline Foam & $\tau_{Y}(\mathrm{~Pa})$ & $k\left(\mathrm{~Pa} \mathrm{~s}^{\beta}\right)$ & $\beta$ \\
\hline Bidisperse foam & $0.4 \pm 0.1$ & $0.9 \pm 0.1$ & $0.40 \pm 0.03$ \\
Monodisperse foam & $0.9 \pm 0.1$ & $0.7 \pm 0.1$ & $0.45 \pm 0.04$ \\
\hline \hline
\end{tabular}

Results of the simulations together with the fits are plotted in Fig. 3, and the fitted parameters are shown in Table II.

The extracted power-law exponent for a bidisperse foam is in good agreement with experiments for a two-dimensional bidisperse foam in both linear and cylindrical shear geometries $[44,49,53,54]$. However, other experiments such as those reported in $[14,17]$ have given significantly smaller values for the exponent. Also three-dimensional foams with low-surface modulus exhibit a similar exponent, as shown in [19]. On the other hand, the yield stress $\tau_{Y}$ is larger than experimental results from $[53,54]$ by a factor of nearly 2 . However, in this case one would expect the model to produce a larger value than the experiments since there is one less spatial degree of freedom present and the actual liquid fraction of the experimental foam is larger than the projected liquid fraction. This result thus provides a magnitude estimate for the difference of yield stress between a true two-dimensional foam and a monolayer of a three-dimensional foam.

In the case of a monodisperse foam the fit to the data was very similar. The yield stress was larger for a monodisperse foam as one would expect as there is more order in the foam. Also, the power-law exponent was slightly larger, again as expected for a more ordered foam, but not near 2/3 as argued in [44] for an ordered monodisperse foam. With the $10 \%$ variation in the bubble diameter, the simulated foam was in fact not truly monodisperse or ordered.

\section{B. Shear-induced pressure}

In addition to the shear stress, we also measured the normal stress, П, exerted by the foam on the confining walls. When sheared, a foam tends to expand, and the resulting increase of pressure in a system with a fixed volume will be related to the shear rate. Motivated by [39], we used a functional form analogous to the Herschel-Bulkley constititive equation (6),

$$
\Pi=\Pi_{0}+c \dot{\gamma}^{\alpha}
$$

where $\Pi_{0}$ is the static osmotic pressure. By fitting again the numerical data, we determined the three parameters of equation Eq. (8). The simulated data together with the fits are shown in Fig. 4, and the fitted parameters are shown in Table III.

For a monodisperse ordered two-dimensional foam the static osmotic pressure can be estimated by the analytical expression

$$
\Pi_{0}=\frac{2 \sigma}{\langle d\rangle} \sqrt{\frac{1-\phi}{1-\phi_{c}}}\left[\sqrt{\frac{\phi_{c}}{\phi}}-1\right] \text {, }
$$




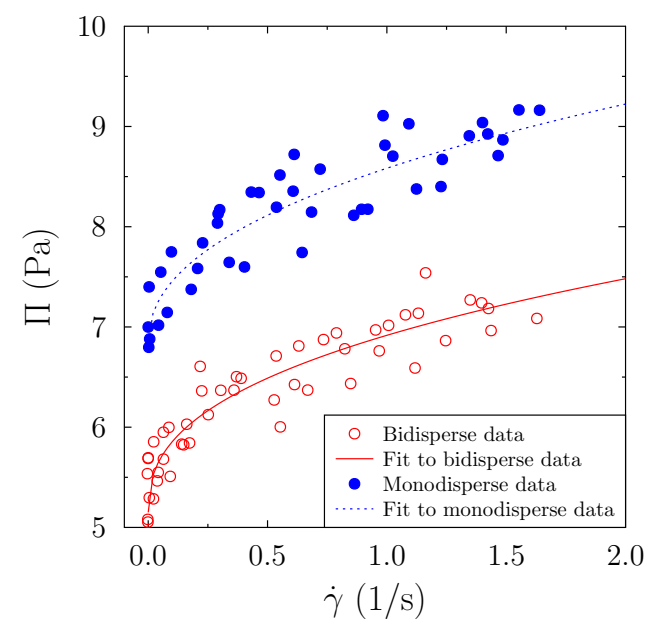

FIG. 4. (Color online) Foam pressure as a function of shear rate for a bidisperse and monodisperse foam. The full and dotted lines are fits to the simulated points (open and filled spheres, respectively) by equation $\Pi=\Pi_{0}+c \dot{\gamma}^{\alpha}$ with coefficients given in Table III.

where $\phi_{c} \approx 0.093$ is the critical liquid fraction for a monodisperse and ordered foam [5,51]. The critical liquid fraction for a polydisperse disordered foam is $\phi_{c} \approx 0.16[2,5,52]$. The liquid fractions of the modeled foams were between $\phi=0.071$, as determined from the nominal bubble volume, and $\phi=0.064$, as determined with the elastic boundary as part of the bubble volume. Equation (9), with $\phi_{c}=0.093$, at $\phi=0.064$ yields $\Pi_{0}=3.8$, which is less than our model. However, this result seems to be consistent with simulations presented in [2] in which the osmotic pressure of a disordered polydisperse foam is found to be larger than the analytical result would suggest.

As for the exponent $\alpha$, we notice that for both foams the fit yields the same values as the corresponding Herschel-Bulkley exponent $\beta$. This implies a linear dependence on the shear stress of the normal stress. So as to illustrate this relationship we combine Eqs. (7) and (8), with the assumption $\alpha=\beta$, which produces the relation

$$
k\left(\Pi-\Pi_{0}\right)=c\left(\tau-\tau_{Y}\right)
$$

for a flowing foam. From this equation, Eq. (10), it is evident that the ratio of pressure to shear stress is constant above the yield stress. However, experimental results from [62] suggest, albeit for quite a different foam, that this might not be the case. This result suggests that, in this respect, the current model is inconsistent with experiments. To narrow down the cause of this inconsistency it would be helpful to know if Eq. (9) is after all a valid description for the normal stress. This issue is,

TABLE III. Best least-squares-fit parameters for the osmotic pressure, $\Pi=\Pi_{0}+c \dot{\gamma}^{\alpha}$, for a bidisperse and monodisperse foam. The errors given correspond to the $1 \sigma$ confidence level.

\begin{tabular}{lccc}
\hline \hline Foam & $\Pi_{0}(\mathrm{~Pa})$ & $c\left(\mathrm{~Pa} \mathrm{~s}^{\alpha}\right)$ & $\alpha$ \\
\hline Bidisperse foam & $5.2 \pm 0.1$ & $1.8 \pm 0.2$ & $0.40 \pm 0.05$ \\
Monodisperse foam & $6.9 \pm 0.2$ & $1.8 \pm 0.3$ & $0.45 \pm 0.09$ \\
\hline
\end{tabular}

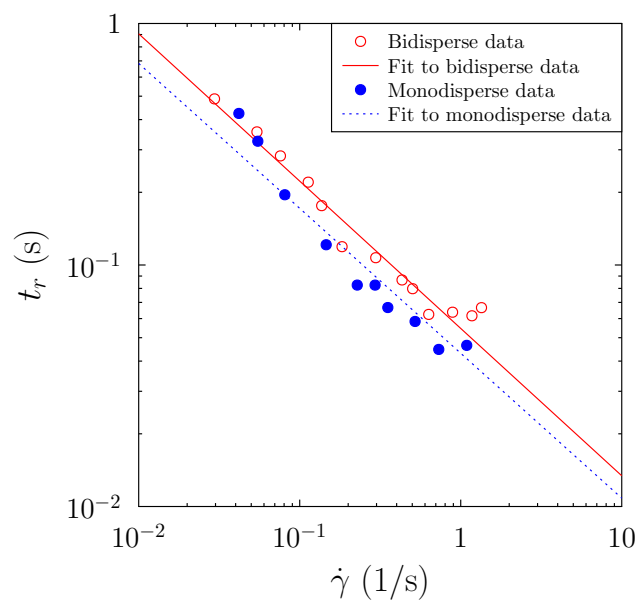

FIG. 5. (Color online) Bubble relaxation time, $t_{r}$, as a function of shear rate, $\dot{\gamma}$, for a bidisperse and monodisperse foam. The full and dotted lines, respectively, correspond to fitting the data by equation $t_{r}=\left(q t_{\text {char }}\right)^{(1-v)} \dot{\gamma}^{-v}$. Fitted coefficients of this equation are shown in Table IV.

however, beyond the scope of the present paper, so we leave it for a forthcoming publication.

\section{Bubble diffusivity}

In [53] a set of measurements were done on bubble diffusion, and a relation

$$
t_{r}=\left(q t_{\mathrm{char}}\right)^{1-v} \dot{\gamma}^{-v}
$$

was discovered between the relaxation time $t_{r}$ and the shear rate $\dot{\gamma}$ of the system. In Eq. (11) $q$ and $v$ are fit parameters, and $t_{\text {char }} \equiv \frac{\mu\langle d\rangle}{\sigma}$ is the characteristic relaxation time of a single bubble. Relaxation time $t_{r}$ is determined by the Lindemann criterion, $s^{2}\left(t=t_{r}\right)=(0.14\langle d\rangle)$, where $s^{2}(t)$ is the mean square non-affine displacement of the bubbles.

From our simulations we could also determine these relaxation times using the same criteria. However, because of the need here to use long time averages, only a few data points could be reliably extracted. Nevertheless, the tentative results based on them are in good agreement with the experimental values reported in [53]. The simulated data together with the curved fitted to them are shown in Fig. 5, and the corresponding fit parameters are shown in Table IV.

Another key result reported in [53] is that the HerschelBulkley exponent, $\beta$, and the relaxation time exponent, $v$, are related so that $\beta \approx 1-v$. Looking up the related values from Tables II and IV, it is evident that we were able to reproduce

TABLE IV. Best least-squares-fit parameters for the relaxation time as a function of shear rate, $t_{r}=\left(q t_{\text {char }}\right)^{(1-v)} \dot{\gamma}^{-v}$, for a bidisperse and monodisperse foam. The errors given correspond to the $1 \sigma$ confidence level.

\begin{tabular}{lcc}
\hline \hline Foam & $q$ & $v$ \\
\hline Bidisperse foam & $3 \pm 3$ & $0.61 \pm 0.05$ \\
Monodisperse foam & $2 \pm 3$ & $0.60 \pm 0.08$ \\
\hline \hline
\end{tabular}


this relationship, within the given errors, for a both bidisperse and monodisperse foam.

\section{Deformations}

Finally we take a look at bubble deformations during shear. We characterize bubble deformations with the ratio $a / b$, where $a$ and $b$ are the semimajor and semiminor axes of an elliptical fit to the bubble. Since the model includes explicitly deformed bubble surfaces, more detailed information about deformations could in principle be retrieved. However, experimentally one is usually constrained to fitting bubbles by ellipses, and for small deformations it is anyway a good approximation.

First we look at the distribution of $a / b-1$ in three cases corresponding to no shear and two different values of shear stress. The related histograms are shown in Fig. 6 for a bidisperse and monodisperse foam. An immediate observation from these histograms is that an average deformation increases and the related distribution broadens as the shear stress is increased.

Comparison to experimental results of [53] shows that deformation distributions of our model are broader than those
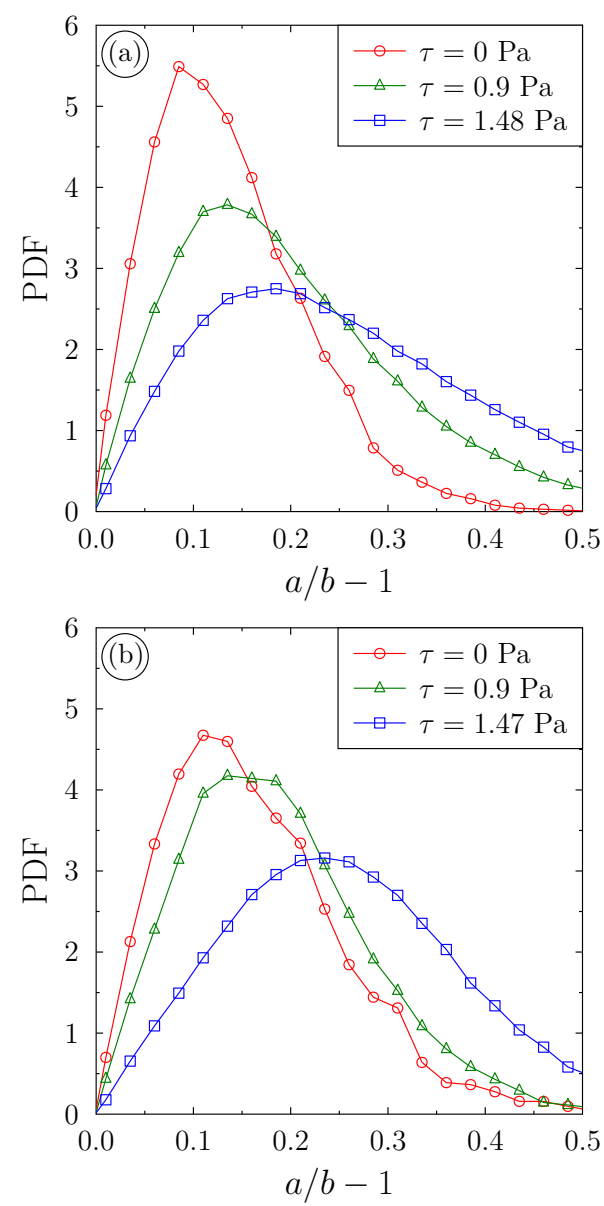

FIG. 6. (Color online) Histograms of bubble deformation in a bidisperse (a) and monodisperse (b) foam characterized by the quantity $a / b-1$, where $a$ and $b$ are the semimajor and semiminor axes of an elliptical fit to the bubble. The three sets correspond to three different values of the shear stress.
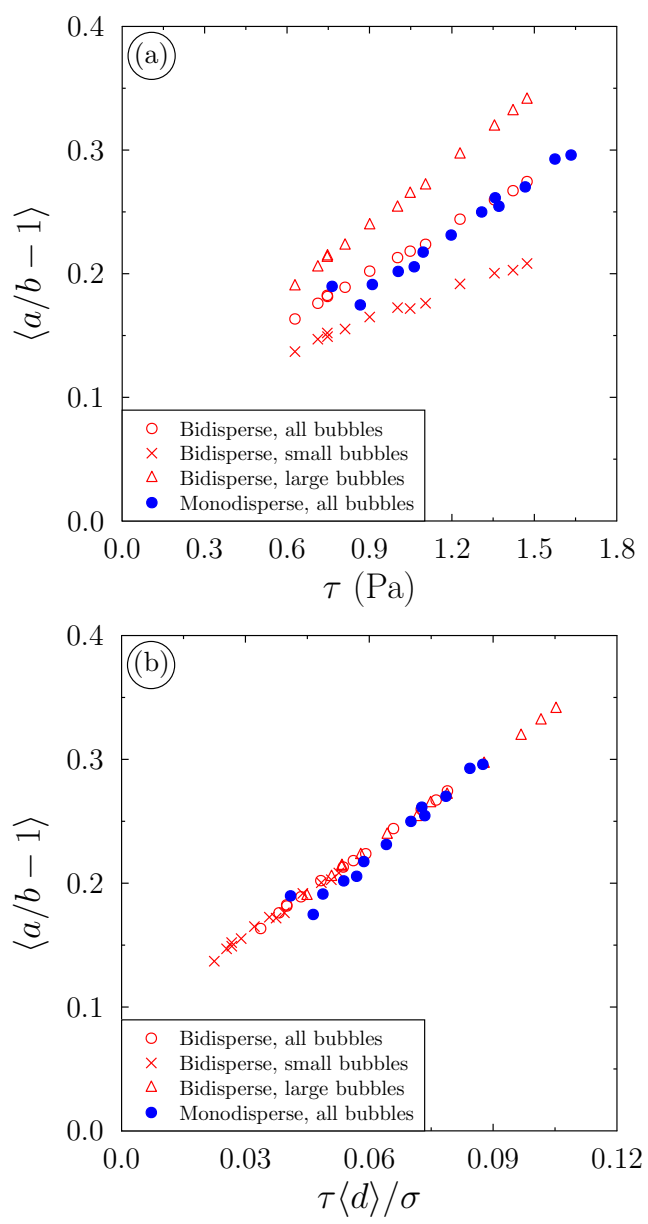

FIG. 7. (Color online) The average deformation of the bubbles as characterized by $a / b-1$, where $a$ and $b$ are the semimajor and semiminor axes, respectively, of an elliptical fit to the bubble, plotted against the shear stress, $\tau$, (a) and the dimensionless shear stress, $\tilde{\tau}=$ $\tau\langle d\rangle / \sigma$ (b). The four data sets correspond to a monodisperse foam, the whole bidisperse foam, and the small-bubble and large-bubble components of the bidisperse foam.

in the experiments. The most obvious explanation to this behavior is that deformations of our model were limited to two dimensions, while in the experiments they were not. When estimating any deformation from its two-dimensional projection, its component in the third dimension will not be observed. In addition, in the experiments bubbles were spheres-but in our model the cylindrical form of the bubbles leads to a different volumetric response when their projected circumference is altered by interactions with neighboring bubbles. However, a more interesting fact is that, as is evident from Fig. 7, we find a clear relationship between the mean bubble deformation and the shear stress (and subsequently the shear rate), while [53] claims that no such trend is observed. We suspect that also this discrepancy is, at least in part, attributed to a difference in the dimensionality of the experiment and the model. In any case, in view of the fact that model reproduces the experimental relationship between the shear rate and shear stress, it is somewhat puzzling that an observable that directly characterizes local geometric aspects of the system does not 
behave similarly in the model and experiment, when there is a reason to believe [38] in particular that the relation of the shear rate and shear stress arises largely from local geometric features of the foam.

\section{SUMMARY AND OUTLOOK}

In this work we introduced the DySMaL model for simulating soft-matter dynamics. We simulated a monolayer of foam exposed to a linear shear, and compared the results with those of recent experiments $[44,49,53,54]$. It was known that slightly simpler wet foam models, such as the Durian disk model [1], have been able to shed light into some of the shear-related phenomena $[4,9,12,39,74]$. In this particular case the DySMaL model quantitatively reproduced experimental observations such as the Herschel-Bulkley power law [Eq. (6)] and the power-law governing the foam relaxation time [Eq. (11)]. This leads us to conclude that the model assumptions are reasonable and the model could be successfully used, in conjunction with other models, to study the microscopic origins of these material properties. Since the possibilities of controlling the microscale are more refined in the DySMaL model than for example in the disk model, we believe that this model is a useful addition to existing foam models. Furthermore, our model suggests relations between bubble deformation, shear stress, and normal stress, which deserve further investigation.

We also found cases of possible discrepancies between the model and experiment, and analyzing these cases may allow further improvements of the model. Of particular interest will, for example, be the functional forms of local forces in the model and how they affect global behavior. We know that the local forces in our model do not strictly adhere to theoretical models like $[19,38,43]$, nevertheless the model works well in a global foam scale. This feature of the model brings forth the question of how sensitive the global behavior is to the actual forms of the local forces, and what are the roles of the global properties such as polydispersity and disorder. We feel that the DySMaL model provides a very agile platform for studying these effects.

Finally, we would like reiterate that in addition to modeling a pure foam, the model is easily modified to include solid objects suspended within the foam. This framework thus opens up the possibility to study a vast set of complex systems of various kind.

\section{ACKNOWLEDGMENTS}

This work was financially supported by the European Regional Development Fund, the Regional Council of Central Finland and the Academy of Finland. Project No. 264427 Computational resources were provided by CSC - IT Center for Science.

\section{APPENDIX A: LAPLACE'S LAW}

The equilibrium condition for the pressure and surface tension of a bubble at rest should be consistent with Laplace's law,

$$
\Delta p=\frac{\sigma}{r}
$$

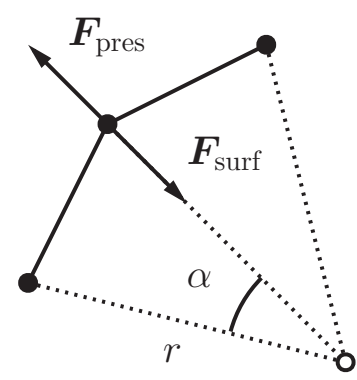

FIG. 8. Pressure and surface-tension forces acting on a particle connecting two segments.

For our discrete description of the bubble surface in terms of segments, the equilibrium condition corresponds to a zero net force on a vertex connecting two segments, which is illustrated in Fig. 8.

According to Eqs. (2) and (3), magnitudes of these forces can be can be expressed in the forms

$$
\left|F_{\text {surf }}\right|=2 \sigma d_{z} \sin \left(\frac{\alpha}{2}\right)
$$

and

$$
\left|F_{\text {pres }}\right|=2 \Delta p r \sin \left(\frac{\alpha}{2}\right) d_{z} \cos \left(\frac{\alpha}{2}\right) .
$$

At equilibrium $\left|F_{\text {surf }}\right|=\left|F_{\text {pres }}\right|$, which gives us

$$
\Delta p=\frac{\sigma}{r \cos \left(\frac{\alpha}{2}\right)}
$$

and since $\alpha=2 \pi / N$ with $N$ the number of segments, we find that

$$
\Delta p=\frac{\sigma}{r \cos \left(\frac{\pi}{N}\right)} .
$$

In the limit $N \rightarrow \infty$ Eq. (A5) satisfies Laplace's law. Already ten segments are sufficient to achieve here a $5 \%$ accuracy.

\section{APPENDIX B: BUOYANCY}

Let us next consider a spherical bubble in a hydrostatic pressure gradient such that $\Delta p(y)=\Delta p_{0}+\rho g y$, where $y$ is the $y$ coordinate. Suppose that this bubble has a radius $r$, it is divided into $N$ segments, and is centered at the origin. Now the net pressure affecting the whole bubble is the sum of pressure contributions of all segments. The $y$ component of the net force on the whole bubble can be determined and it is given by

$$
\begin{aligned}
\sum_{i}^{N} F_{\text {pres }_{y}}= & \sum_{i}^{N}\left[\left(\Delta p_{0}+\rho g r \sin \left(\frac{2 \pi}{N} i\right)\right)\right. \\
& \left.\times r \sin \left(\frac{2 \pi}{N}\right) d_{z} \sin \left(\frac{2 \pi}{N} i\right)\right] \\
= & \frac{\rho g d_{z} r^{2}}{2} N \sin \left(\frac{2 \pi}{N}\right) \stackrel{N \rightarrow \infty}{\longrightarrow} \rho g d_{z} \pi r^{2},
\end{aligned}
$$

while its $x$ component is trivially zero. It is evident that in the $N \rightarrow \infty$ limit Eq. (B1) agrees with the buoyancy of a cylindrical object in a hydrostatic pressure gradient. Again, the accuracy for a ten-segment bubble is roughly $7 \%$. 
[1] D. J. Durian, Phys. Rev. Lett. 75, 4780 (1995).

[2] S. Hutzler and D. Weaire, J. Phys.: Condensed Matter 7, L657 (1995).

[3] T. G. Mason, M.-D. Lacasse, G. S. Grest, D. Levine, J. Bibette, and D. A. Weitz, Phys. Rev. E 56, 3150 (1997).

[4] S. Tewari, D. Schiemann, D. J. Durian, C. M. Knobler, S. A. Langer, and A. J. Liu, Phys. Rev. E 60, 4385 (1999).

[5] D. Weaire and S. Hutzler, The Physics of Foams (Clarendon Press, Oxford, 1999).

[6] G. Debrégeas, H. Tabuteau, and J.-M. di Meglio, Phys. Rev. Lett. 87, 178305 (2001).

[7] A. J. Webster and M. E. Cates, Langmuir 17, 595 (2001).

[8] M. Rosa, M. Fortes, and M. Vaz, Eur. Phys. J. E 7, 129 (2002).

[9] I. K. Ono, C. S. O’Hern, D. J. Durian, S. A. Langer, A. J. Liu, and S. R. Nagel, Phys. Rev. Lett. 89, 095703 (2002).

[10] J. Lauridsen, M. Twardos, and M. Dennin, Phys. Rev. Lett. 89, 098303 (2002).

[11] I. Cantat and R. Delannay, Phys. Rev. E 67, 031501 (2003).

[12] I. K. Ono, S. Tewari, S. A. Langer, and A. J. Liu, Phys. Rev. E 67, 061503 (2003).

[13] A. Kabla and G. Debrégeas, Phys. Rev. Lett. 90, 258303 (2003).

[14] E. Pratt and M. Dennin, Phys. Rev. E 67, 051402 (2003).

[15] N. Kern, D. Weaire, A. Martin, S. Hutzler, and S. J. Cox, Phys. Rev. E 70, 041411 (2004).

[16] Q. Sun and S. Hutzler, Rheologica Acta 43, 567 (2004).

[17] J. Lauridsen, G. Chanan, and M. Dennin, Phys. Rev. Lett. 93, 018303 (2004).

[18] I. Cohen, T. G. Mason, and D. A. Weitz, Phys. Rev. Lett. 93, 046001 (2004).

[19] N. D. Denkov, V. Subramanian, D. Gurovich, and A. Lips, Colloids and Surfaces A: Physicochemical and Engineering Aspects 263, 129 (2005), a collection of papers presented at the 5th European Conference on Foams, Emulsions, and Applications, \{EUFOAM 2004, University of Marne-la-Vallee, Champs sur Marne (France), 5-8 July, 2004.

[20] R. Höhler and S. Cohen-Addad, J. Phys.: Condensed Matter 17, R1041 (2005).

[21] Q. Sun and S. Hutzler, Colloids and Surfaces A: Physicochemical and Engineering Aspects 263, 27 (2005).

[22] R. J. Clancy, E. Janiaud, D. Weaire, and S. Hutzler, Eur. Phys. J. E: Soft Matter 21, 123 (2006).

[23] M. Durand and H. A. Stone, Phys. Rev. Lett. 97, 226101 (2006).

[24] E. Janiaud, D. Weaire, and S. Hutzler, Phys. Rev. Lett. 97, 038302 (2006).

[25] A. Saugey, W. Drenckhan, and D. Weaire, Phys. Fluids 18, 053101 (2006).

[26] E. Terriac, J. Etrillard, and I. Cantat, EPL 74, 909 (2006).

[27] Y. Wang, K. Krishan, and M. Dennin, Phys. Rev. E 73, 031401 (2006).

[28] Y. Wang, K. Krishan, and M. Dennin, Phys. Rev. E 74, 041405 (2006).

[29] A. Kabla and G. Debregeas, Journal of Fluid Mechanics 587, 23 (2007).

[30] A. Kabla, J. Scheibert, and G. Debregeas, Journal of Fluid Mechanics 587, 45 (2007).

[31] S. Cohen-Addad, M. Krzan, R. Höhler, and B. Herzhaft, Phys. Rev. Lett. 99, 168001 (2007).

[32] B. Dollet and F. Graner, Journal of Fluid Mechanics 585, 181 (2007).
[33] Q. Sun, W. Ge, and J. Huang, Chinese Science Bulletin 52, 423 (2007).

[34] V. Labiausse, R. Hhler, and S. Cohen-Addad, J. Rheol. 51, 479 (2007).

[35] P. Marmottant and F. Graner, Eur. Phys. J. E: Soft Matter 23, 337 (2007).

[36] S. Besson, G. Debrégeas, S. Cohen-Addad, and R. Höhler, Phys. Rev. Lett. 101, 214504 (2008).

[37] I. Cheddadi, P. Saramito, C. Raufaste, P. Marmottant, and F. Graner, Eur. Phys. J. E 27, 123 (2008).

[38] N. D. Denkov, S. Tcholakova, K. Golemanov, K. P. Ananthapadmanabhan, and A. Lips, Phys. Rev. Lett. 100, 138301 (2008).

[39] V. J. Langlois, S. Hutzler, and D. Weaire, Phys. Rev. E 78, 021401 (2008).

[40] B. Markert, Archives of Computational Methods in Engineering 15, 371 (2008).

[41] P. Rognon and C. Gay, Eur. Phys. J. E 27, 253 (2008).

[42] R. Soller and S. A. Koehler, Phys. Rev. Lett. 100, 208301 (2008).

[43] S. Tcholakova, N. D. Denkov, K. Golemanov, K. P. Ananthapadmanabhan, and A. Lips, Phys. Rev. E 78, 011405 (2008).

[44] G. Katgert, M. E. Möbius, and M. van Hecke, Phys. Rev. Lett. 101, 058301 (2008).

[45] D. Weaire, S. Hutzler, V. Langlois, and R. Clancy, Philosophical Magazine Letters 88, 387 (2008).

[46] N. D. Denkov, S. Tcholakova, K. Golemanov, K. P. Ananthpadmanabhan, and A. Lips, Soft Matter 5, 3389 (2009).

[47] P. Rognon and C. Gay, Eur. Phys. J. E 30, 291 (2009).

[48] B. Embley and P. Grassia, Colloids and Surfaces A: Physicochemical and Engineering Aspects 344, 24 (2009), current research on foams.

[49] G. Katgert, A. Latka, M. E. Möbius, and M. van Hecke, Phys. Rev. E 79, 066318 (2009).

[50] C. Raufaste, A. Foulon, and B. Dollet, Phys. Fluids 21, 053102 (2009).

[51] W. Drenckhan and D. Langevin, Current Opinion in Colloid \& Interface Science 15, 341 (2010).

[52] G. Katgert and M. van Hecke, EPL 92, 34002 (2010).

[53] M. E. Möbius, G. Katgert, and M. van Hecke, EPL 90, 44003 (2010).

[54] G. Katgert, B. P. Tighe, M. E. Möbius, and M. van Hecke, EPL 90, 54002 (2010).

[55] C. Raufaste, S. J. Cox, P. Marmottant, and F. Graner, Phys. Rev. E 81, 031404 (2010).

[56] B. P. Tighe, E. Woldhuis, J. J. C. Remmers, W. van Saarloos, and M. van Hecke, Phys. Rev. Lett. 105, 088303 (2010).

[57] P. Rognon, I. Einav, and C. Gay, Phys. Rev. E 81, 061304 (2010).

[58] M. Durand, J. Käfer, C. Quilliet, S. Cox, S. A. Talebi, and F. Graner, Phys. Rev. Lett. 107, 168304 (2011).

[59] S. Jones, B. Dollet, N. Slosse, Y. Jiang, S. Cox, and F. Graner, Colloids and Surfaces A: Physicochemical and Engineering Aspects 382, 18 (2011).

[60] B. P. Tighe, Phys. Rev. Lett. 107, 158303 (2011).

[61] S. Hutzler and D. Weaire, Colloids and Surfaces A: Physicochemical and Engineering Aspects 382, 3 (2011), a collection of papers from the 8th $\{$ EUFOAM $\}$ Conference and the Meetings of $\{$ COST $\}$ Actions $\{$ D43 $\}$ and $\{$ P21 $\}$.

[62] R. Lespiat, S. Cohen-Addad, and R. Höhler, Phys. Rev. Lett. 106, 148302 (2011). 
[63] P. Rognon, I. Einav, and C. Gay, Journal of Fluid Mechanics 689, 75 (2011).

[64] P. Ballesta, G. Petekidis, L. Isa, W. C. K. Poon, and R. Besseling, Journal of Rheology 56, 1005 (2012).

[65] I. Cheddadi, P. Saramito, and F. Graner, Journal of Rheology 56, 213 (2012).

[66] I. Fortuna, G. L. Thomas, R. M. C. de Almeida, and F. Graner, Phys. Rev. Lett. 108, 248301 (2012).

[67] M. Le Merrer, S. Cohen-Addad, and R. Höhler, Phys. Rev. Lett. 108, 188301 (2012).

[68] H. Mohammadigoushki and J. J. Feng, Phys. Rev. Lett. 109, 084502 (2012).

[69] P. Olsson and S. Teitel, Phys. Rev. Lett. 109, 108001 (2012).
[70] C. B. ODonovan, E. I. Corwin, and M. E. Möbius, Philosophical Magazine 93, 4030 (2013).

[71] M. Kogan, L. Duclou, J. Goyon, X. Chateau, O. Pitois, and G. Ovarlez, Rheologica Acta 52, 237 (2013).

[72] C.-C. Kuo and M. Dennin, Phys. Rev. E 87, 030201 (2013).

[73] M. Wyart and M. E. Cates, Phys. Rev. Lett. 112, 098302 (2014).

[74] M. Sexton, S. Hardiman, M. Möbius, and S. Hutzler, Physica A: Statistical Mechanics and its Applications 394, 312 (2014).

[75] See Supplemental Material at http://link.aps.org/supplemental/ 10.1103/PhysRevE.90.032307 for a video illustration of the simulation.

[76] A. Koponen and A. Jäsberg (private communication). 